J. Phys. IV France 138 (2006) 237-244

(C) EDP Sciences, Les Ulis

DOI: $10.1051 /$ jp4:2006138027

\title{
Optiques multicouches EUV
}

\author{
J. Gautier ${ }^{1}$, F. Delmotte ${ }^{1}$, F. Bridou ${ }^{1}$, M. Rouillay ${ }^{2}$, F. Varniere ${ }^{1}$, S. de Rossi ${ }^{1}$, \\ A. Jerome ${ }^{1}$ et M.F. Ravet ${ }^{1}$ \\ ${ }^{1}$ Laboratoire Charles Fabry de I'Institut d'Optique, CNRS-UMR 8501, Bât. 503, \\ Centre Scientifique d'Orsay, 91403 Orsay, France \\ 2 LIXAM, Bât. 350, Université Paris Sud, 91405 Orsay, France
}

\begin{abstract}
Résumé. L'élaboration de nouveaux revêtements interférentiels multicouches est motivée par la demande de l'astrophysique solaire et par le développement de nouvelles sources de rayonnement EUV (source à génération d'harmoniques d'ordre élevé, laser $\mathrm{X}$, rayonnement synchrotron). Nous avons étudié et développé différents systèmes multicouches possédant à la fois des propriétés optiques optimisées (réflectivité et/ou bande passante) et une bonne stabilité temporelle et thermique pour la gamme de longueurs d'onde comprise entre $\lambda=30 \mathrm{~nm}$ et $\lambda=50 \mathrm{~nm}$. Une augmentation significative de la réflectivité des multicouches dans la gamme spectrale de 30 à $40 \mathrm{~nm}$ a été obtenue par l'addition d'un troisième matériau dans l'empilement. Une étude comparative des courbes de réflectivités théorique et expérimentale en fonction de la longueur d'onde a été menée. Les différences observées ont pu être expliquées notamment à l'aide de l'analyse des propriétés physiques des différents matériaux en couche mince. Nous montrons également que l'utilisation de ces multicouches à trois matériaux par période permet le développement d'empilements répondant à des demandes spécifiques (miroirs à large bande passante, miroirs à double bande). Pour les longueurs d'ondes comprises entre $35 \mathrm{~nm}$ et $50 \mathrm{~nm}$ nous avons développé des multicouches à base de scandium. Cette étude a permis d'obtenir des multicouches avec de forts pouvoirs réflecteurs et une bonne stabilité temporelle. La stabilité de ces empilements a pu être améliorée à l'aide de l'insertion aux interfaces de couches barrières.
\end{abstract}

\section{INTRODUCTION}

La région de longueurs d'onde EUV, comprise entre le rayonnement $\mathrm{X}$ dur et le rayonnement ultraviolet offre de grandes possibilités scientifiques et technologiques. Ces courtes longueurs d'onde, typiquement comprises entre $3 \mathrm{~nm}$ et $60 \mathrm{~nm}$, permettent par exemple le diagnostic de plasma dense ou la gravure de motifs nanométriques. Les miroirs interférentiels multicouches permettent d'utiliser ce rayonnement à des angles d'incidences proches de la normale avec des forts pouvoirs réflecteur. Le développement de ces miroirs est fortement lié au développement des nouvelles sources EUV (rayonnement synchrotron, laser X, génération d'harmoniques) et de leurs applications (lithographie EUV, diagnostics de plasma dense).

Le présent travail porte sur l'étude et l'amélioration des miroirs interférentiels multicouches dans la gamme de longueurs d'onde comprise entre $30 \mathrm{~nm}$ et $50 \mathrm{~nm}$ Après une brève description des dispositifs de dépôt et de caractérisations utilisés, nous présenterons tout d'abord une étude des performances et de la stabilité de multicouches à base de scandium pour les longueurs d'onde supérieures à $30 \mathrm{~nm}$. Puis nous montrerons ensuite que l'utilisation d'un troisième matériau dans un empilement périodique peut augmenter la réflectivité. Une étude comparative entre les réflectivités expérimentales et théoriques sera proposée. Enfin nous présenterons deux applications réalisées à l'aide de ces multicouches à trois matériaux par période. 


\section{SYSTÈME DE DÉPÔT ET MOYENS DE CARACTÉRISATIONS}

\subsection{Système de dépôt}

Les multicouches développées sont déposées à l'aide d'un réacteur de dépôt par pulvérisation cathodique magnétron. La pulvérisation cathodique est un procédé basé sur la pulvérisation d'une cible solide à partir d'un plasma généré dans un gaz à base pression. Le schéma du système de dépôt, implanté dans la salle blanche du LCFIO, est représenté sur la figure 1 . Ce système possède 4 cibles de dimension $80 \times 200 \mathrm{~mm}^{2}$ montées dans le fond de l'enceinte. Une pression inférieure à $10^{-8}$ Torr est obtenue à l'aide d'une pompe cryogénique. Les différentes couches minces sont déposées par passages successifs de l'échantillon au dessus des cibles à une distance de $10 \mathrm{~cm}$. L'épaisseur de chaque couche est contrôlée par la vitesse de rotation de l'échantillon au dessus des cibles. Le contrôle de cette vitesse permet d'obtenir une uniformité des dépôts inférieur à $1 \%$ sur un diamètre de $125 \mathrm{~mm}$.

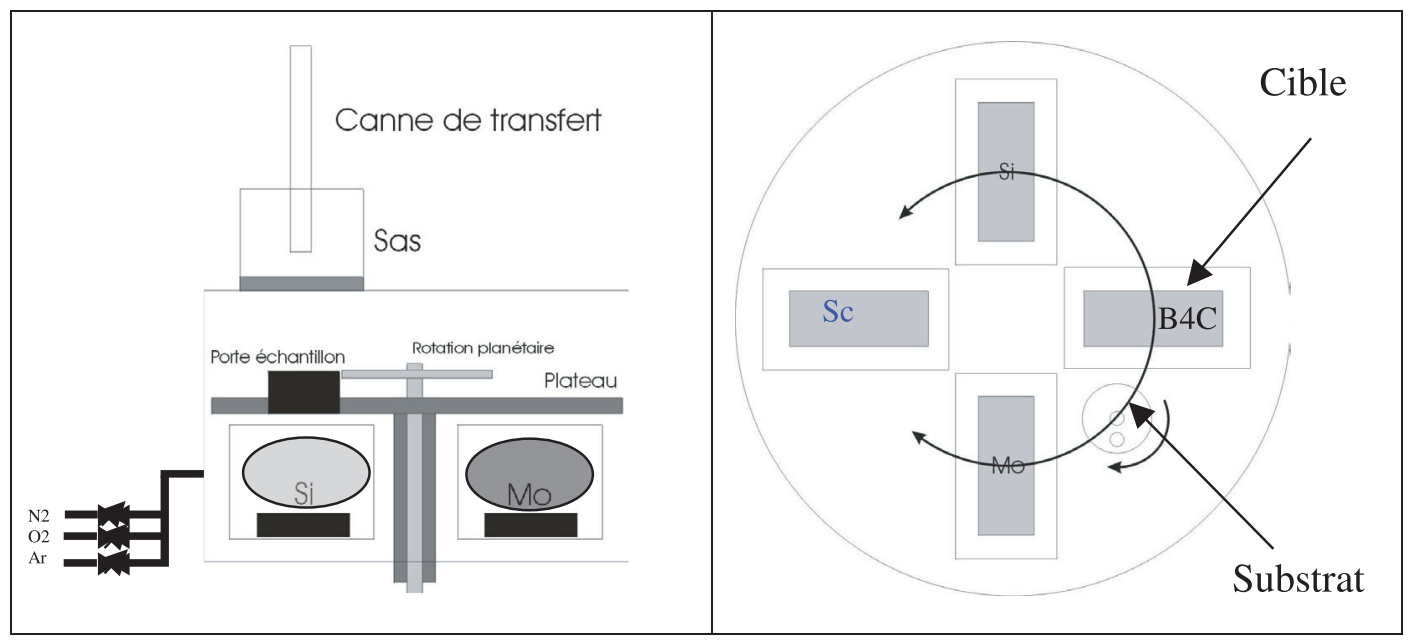

Figure 1. Schéma du réacteur de pulvérisation cathodique magnétron.

\subsection{Moyens de caractérisations}

La compréhension des systèmes multicouches nécessite l'étude physico-chimique et structurale des couches composant l'empilement. Apres chaque dépôt, les multicouches sont analysées par réflectométrie en $\mathrm{X}$ rasant à $\lambda=0.154 \mathrm{~nm}$. La résolution angulaire du réflectométre est meilleure que 0.001 degré. L'ajustement de la courbe expérimentale à permis de déterminer la période de l'empilement, l'épaisseur des différentes couches, l'indice complexe des matériaux à cette longueur d'onde ainsi que la rugosité aux interfaces [1].

Pour caractériser les multicouches à la longueur d'onde d'utilisation, nous avons mesuré les réflectivités des multicouches à l'aide du réflectométre de la ligne BEAR du rayonnement synchrotron d'Elletra sous un angle d'incidence de $10^{\circ}$ en polarisation S [2].

L'analyse par rétrodiffusion coulombienne et l'analyse nucléaire nous a permis de déterminer la densités et les impuretés présentes dans les couches minces composant l'empilement. Le tableau 1 dresse le bilan des différentes caractéristiques mesurées.

Tableau 1. Caractéristiques mesurées des couches minces utilisées.

\begin{tabular}{|c|c|c|c|c|}
\hline & Scandium & Molybdène & Silicium & Carbure de bore \\
\hline Densité expérimentale & $2.8 \mathrm{~g} \mathrm{~cm}^{-3}$ & $10.1 \mathrm{~g} \mathrm{~cm}^{-3}$ & $2.1 \mathrm{~g} \mathrm{~cm}^{-3}$ & $1.8 \mathrm{~g} \mathrm{~cm}^{-3}$ \\
\hline Densité théorique & $2.9 \mathrm{~g} \mathrm{~cm}^{-3}$ & $10.2 \mathrm{~g} \mathrm{~cm}^{-3}$ & $2.2 \mathrm{~g} \mathrm{~cm}^{-3}$ & $2.5 \mathrm{~g} \mathrm{~cm}^{-3}$ \\
\hline Impuretés & Fer $(430 \mathrm{ppm})$ Tantale $(<1000 \mathrm{ppm})$ & - & $4 \% \mathrm{Ar}$ & $3 \% \mathrm{Ar}$ \\
\hline
\end{tabular}




\section{MULTICOUCHES SCANDIUM/SILICIUM DANS LA GAMME SPECTRALE 40-50 NM}

De nombreuses applications nécessitent des miroirs réflecteurs en incidence quasi-normale dans la gamme de longueurs d'onde comprise entre $40 \mathrm{~nm}$ et $50 \mathrm{~nm}$. C'est le cas par exemple des lasers à décharges capillaires et des sources à générations d'harmoniques. L'imagerie spatiale solaire, pour la raie Ne VII $(\lambda=46.5 \mathrm{~nm})$ est aussi intéressée par des revêtements à fort pouvoir réflecteur. A partir de calculs théoriques Uspenskii et al ont montré dés 1998 que les empilements à base de scandium semblent être de bons candidats pour cette gamme de longueur d'ondes [3]. Deux équipes ont réalisé et mesuré des multicouches $\mathrm{Sc} / \mathrm{Si}$ [4] [5]. La réflectivité de ces multicouches est prometteuse, mais elles présentent une mauvaise stabilité. Nous avons donc étudié ces multicouches et cherché à améliorer la stabilité.

\subsection{Performances des multicouches $\mathrm{Sc} / \mathrm{Si}$}

Nous avons développé plusieurs systèmes multicouches $\mathrm{Sc} / \mathrm{Si}$. Sur la figure 2 sont représentées la réflectivité mesurée d'une multicouche centrée à $45.6 \mathrm{~nm}$ ainsi que la réflectivité calculée à partir de la période de la multicouche. Nous avons mesuré une réflectivité élevée de $37 \%$ à $45.6 \mathrm{~nm}$. Nous avons aussi réalisé des multicouches avec des pouvoirs réflecteurs de $22 \%$ à $40.6 \mathrm{~nm}$ et de $34 \%$ à $48.4 \mathrm{~nm}$. Cependant nous avons constaté une grande différence entre la réflectivité théorique et expérimentale. Cette différence est due aux propriétés physico-chimiques des couches composant l'empilement. En effet lors des micro-analyses nucléaires de films minces nous avons constaté la présence de fer et de tantale dans la couche de scandium. Cependant des simulations tenant compte de ces impuretés ont montré qu'elles ne sont pas responsables d'une chute significative de la réflectivité. La présence d'argon dans la couche de silicium peut diminuer la réflectivité. En effet $4 \%$ d'argon diminue relativement la réflectivité de $20 \%$. Cependant cette chute n'est pas suffisante pour expliquer cette différence entre les deux courbes. La formation d'oxyde de silicium en surface est la principale cause de la chute de réflectivité. Comme nous pouvons le voir sur la figure 2, $2 \mathrm{~nm}$ d'oxyde de silicium permet d'obtenir un bon accord antre la réflectivité théorique et expérimentale. Pour augmenter la réflectivité expérimentale nous avons optimisé la dernière couche de silicium. Une dernière couche de silicium d'épaisseur de $3.2 \mathrm{~nm}$ (au lieu de $12.8 \mathrm{~nm}$ dans l'empilement), permet d'obtenir une réflectivité élevée de $46 \%$ à $45.6 \mathrm{~nm}$. Une réflectivité identique a été obtenue en terminant l'empilement par une couche de carbure de bore de $2 \mathrm{~nm}$.

\subsection{Stabilité temporelle et thermique des multicouches Sc/Si}

La stabilité temporelle des systèmes multicouches est un paramètre important pour de nombreuses applications. La stabilité temporelle a été étudiée par le suivi dans le temps (4 mesures échelonnées) de la réflectivité d'un même échantillon mesurée au rayonnement synchrotron sous une incidence de $10^{\circ}$. Entre les mesures la multicouche était stockée à l'air. Une réflectivité supérieure à $34 \%$ à $\lambda=46 \mathrm{~nm}$ a été mesurée quatorze mois après la fabrication de la multicouche. Nous n'avons pas observé non plus de décalage du pic de réflectivité durant cette période.

Certaines applications comme les optiques pour l'imagerie solaire ou des sources à fort flux demandent une stabilité thermique des revêtements multicouches. Sur la figure 3 sont représentées les réflectivités en fonction de la longueur d'onde d'une multicouche $\mathrm{Sc} / \mathrm{Si}$ avant (ligne) et après un recuit rapide d'une minute à $200^{\circ} \mathrm{C}$ sous Argon. On peut remarquer que le pic de réflectivité se décale de $1.3 \mathrm{~nm}$ et que la réflectivité maximum chute de $4 \%$. Cette faible stabilité thermique peut s'expliquer par la formation d'un composé à l'interface entre le scandium et le silicium, l'épaisseur de celui-ci augmentant avec la température. Deux solutions proposées dans la littérature pour améliorer cette stabilité sont l'ajout de couches barrières de W [6] ou de $\mathrm{Cr}$ [7]. Cependant, l'ajout de ces couches barrières se fait au détriment de la réflectivité. Le but de notre étude est de développer une structure $\mathrm{Sc} / \mathrm{X} / \mathrm{Si} / \mathrm{X}$ où $\mathrm{X}$ 


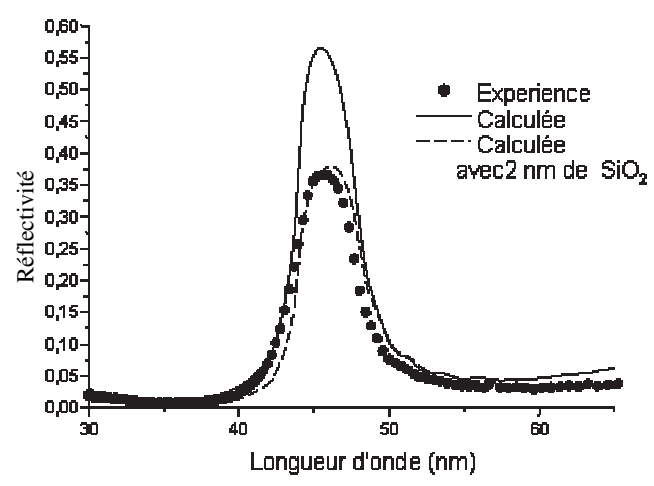

Figure 2. Réflectivités expérimentale et calculées d'une multicouche $\mathrm{Sc} / \mathrm{Si}$ en fonction de la longueur d'onde.

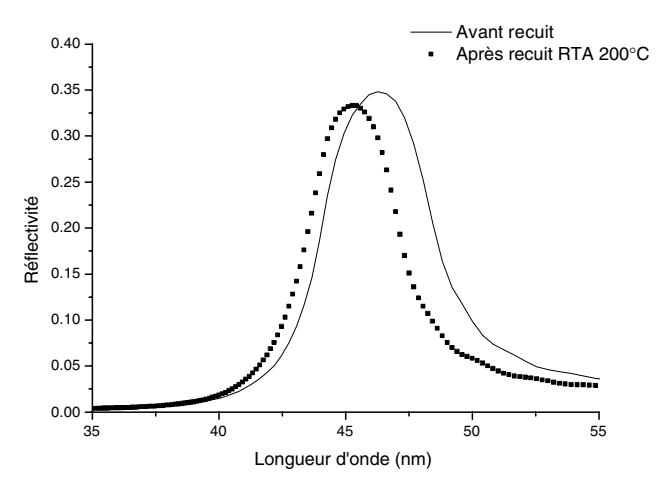

Figure 3. Réflectivités en fonction de la longueur d'onde d'une multicouche $\mathrm{Sc} / \mathrm{Si}$ avant (ligne) et après un recuit rapide d'une minute à $200^{\circ} \mathrm{C}$ sous Argon.

représente la couche barrière, permettant d'améliorer la stabilité thermique de la structure en conservant la réflectivité initiale. Pour cela nous avons comparé les réflectivités obtenues avec et sans barrière avant et après recuit. Les matériaux que nous avons utilisé comme couches barrières sont $\mathrm{B} 4 \mathrm{C}$ et $\mathrm{ScN}$. Nous avons étudié trois épaisseurs différentes : $0.3 \mathrm{~nm}, 0.9$ et $1.5 \mathrm{~nm}$. Les recuits thermiques ont été réalisés à l'aide d'un four à recuit rapide pendant une minute à $200^{\circ} \mathrm{C}$ sous Argon. Les réflectivités des multicouches avant (traits pleins) et après recuit (traits pointillés) sont pour les différentes épaisseurs représentées sur la figure 4. Nous pouvons constater que la réflectivité diminué quand l'épaisseur de la couche barrière augmente : cette diminution est causé par l'absorption. La réflectivité maximale des multicouches avec barrières après recuit est diminuée, la variation de réflectivité avant et après recuit est moins importante pour les multicouches ayant des barrières d'interdiffusion que pour celles sans les barrières. Cependant, une augmentation de l'épaisseur de la barrière ne diminue pas la différence entre la réflectivité maximale avant et après recuit. La variation de réflectivité est minimale pour une épaisseur de $0,3 \mathrm{~nm}$ pour les deux types de barrière. La variation est toujours plus importante quand la barrière est en $\mathrm{ScN}$ qu'en $\mathrm{B}_{4} \mathrm{C}$.

Nous pouvons aussi constater que le décalage du pic de réflectivité avant et après recuit est moins important quand l'épaisseur de la barrière augmente. Ce décalage est de 2,1\% sans barrière, ce qui représente un décalage de $1 \mathrm{~nm}$ en longueur d'onde, il est de $0,7 \%$ avec les barrières de $0,3 \mathrm{~nm}$.

Nous pouvons donc conclure qu'une barrière de $0.3 \mathrm{~nm}$ de $\mathrm{B}_{4} \mathrm{C}$ permet d'avoir un bon compromis entre une bonne stabilité thermique et une réflectivité élevée.

\section{MULTICOUCHES À TROIS MATÉRIAUX ET APPLICATIONS}

Nous avons développé et étudié des systèmes multicouches à trois matériaux [8]. Ces systèmes $\mathrm{B}_{4} \mathrm{C} / \mathrm{Mo} / \mathrm{Si}$ permettent d'augmenter significativement la réflectivité. Une réflectivité expérimentale de $34 \%$ à $\lambda=32 \mathrm{~nm}$ ce qui représente une augmentation importante par rapport à une multicouche classique de type $\mathrm{B}_{4} \mathrm{C} / \mathrm{Si}$. De plus la bande passante d'un système à trois matériaux par période est plus importante. C'est pourquoi nous avons décidé d'utiliser de tels systèmes pour développer deux multicouches spécifiques : une multicouche à large bande passante et une multicouche présentant deux pics de réflectivité.

\subsection{Multicouches à large bande passante}

Certaines applications nécessitent des bandes passantes spectrales ou/et angulaires larges. C'est le cas, par exemple, lors de l'utilisation de sources à spectre large pour lesquelles le maximum de réflectivité 


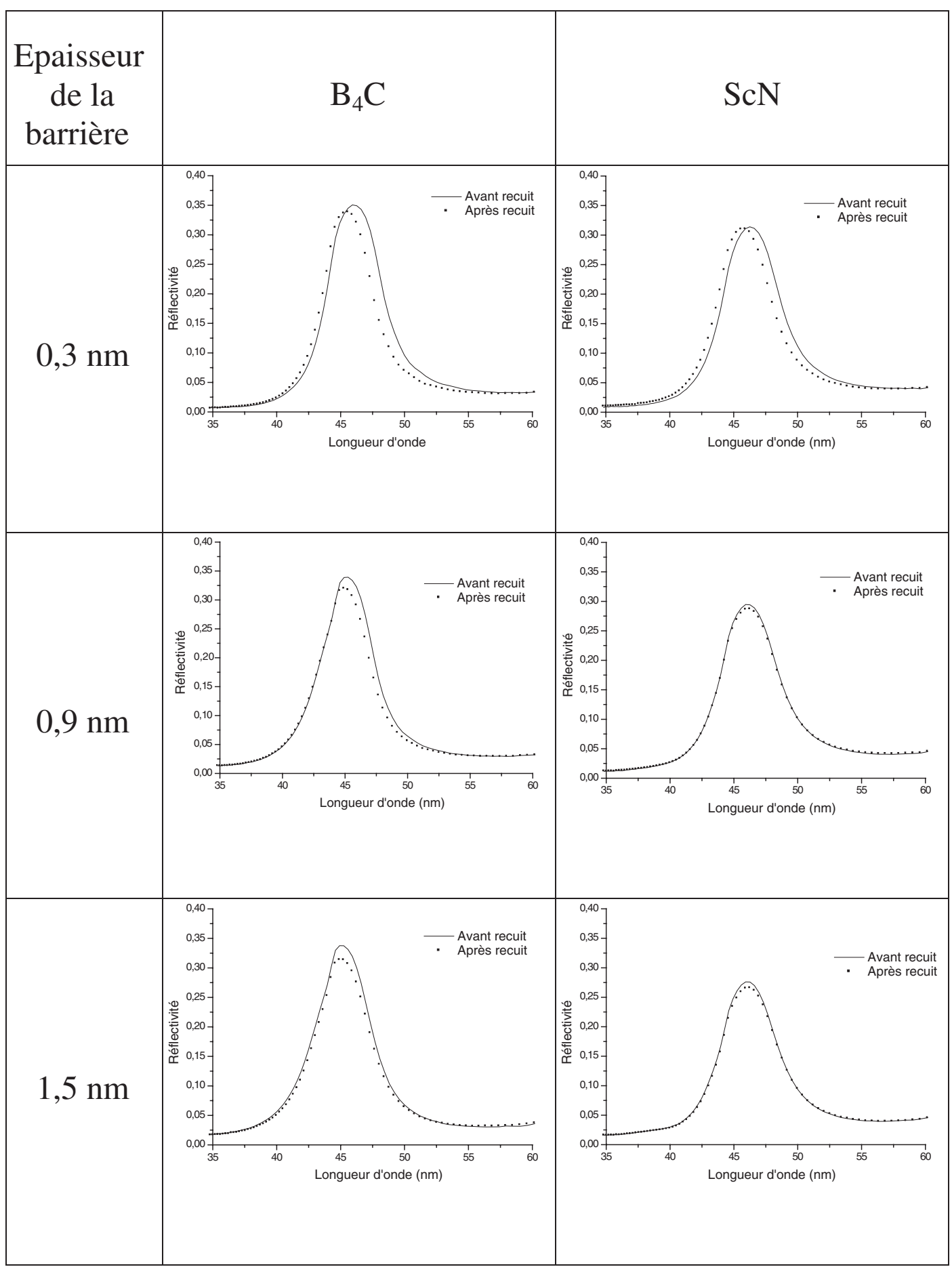

Figure 4. Réflectivités expérimentales en fonction de la longueur d'onde pour des multicouches $\mathrm{Sc} / \mathrm{Si}$ avec des barrières en $\mathrm{B}_{4} \mathrm{C}$ et $\mathrm{ScN}$ de différentes épaisseurs. 
intégrée est désiré (lithographie) ou dans le cas où plusieurs angles d'incidence peuvent être réfléchis (miroirs collecteurs). C'est aussi le cas dans les applications de spectroscopie utilisant des réseaux multicouches [9]. Pour réaliser cette multicouche nous avons superposé deux multicouches périodiques à trois matériaux par période. Nous avons optimisé chaque épaisseur des matériaux à l'aide d'un code développé sous Matlab. Le nombre de période est de 4 pour la multicouche se trouvant sur le substrat. La multicouche de dessus possède 2 périodes. La courbe de réflectivité expérimentale est représenté sur la figure 5 (a). Nous avons mesuré une bande spectrale de $12.6 \mathrm{~nm}$ avec une réflectivité moyenne proche de $17 \%$ entre 25 et $35 \mathrm{~nm}$. Ces résultats sont proches des valeurs théoriques (respectivement $14 \mathrm{~nm}$ et 19\%). Cependant, nous pouvons constater que la forme de courbe n'est pas la même. Cette différence de forme est sans doute du à une erreur de calibration d'un des matériaux.

Cette multicouche a été utilisée dans un montage avec source à génération d'harmoniques permettant la génération d'impulsions sub-femtoseconde. La génération d'impulsion attoseconde a été démontrée par Paul et al. [10]. Cependant un déphasage intrinsèque existe entre les différentes harmoniques qui augmente la durée de l'impulsion [11]. L'objectif de ces miroirs multicouches est de permettre à de nombreuses harmoniques d'être réfléchies sur l'optique et de compenser le déphasage intrinsèque entre les harmoniques [12]. En effet, un miroir multicouche déphase l'onde incidente. Ce déphasage dépend de la longueur d'onde. L'empilement mesuré n'a pas été optimisé pour produire le déphasage souhaité mais il permet la réflexion de plusieurs harmoniques (au moins quatre) et introduit un déphasage. La mesure du déphase de ce miroir en fonction de l'énergie, réalisée à l'aide de la méthode RABBIT, est représentée sur la figure 5 (b). Nous pouvons constater, comme dans le cas de la réflectivité, une grande différence entre le déphasage théorique et expérimental. Cependant le déphasage mesuré compense un peu le déphasage intrinsèque entre harmoniques et laisse espérer une compression de l'impulsion.

\subsection{Multicouches double bande}

Nous avons étudié la possibilité de réaliser un miroir réfléchissant deux longueurs d'onde particulières. Ce miroir aurait l'intérêt de permettre l'observation de deux raies caractéristiques de la couronne solaire, en couplant au télescope un jeu de filtre sélectionnant successivement chacune des deux longueurs d'onde. Notre approche consiste à optimiser une structure composée de deux multicouches périodiques superposées dans le but de filtrer efficacement les deux longueurs d'onde d'intérêt. Pour montrer la faisabilité de ce principe, nous avons choisi deux raies du spectre solaire dans la gamme XUV
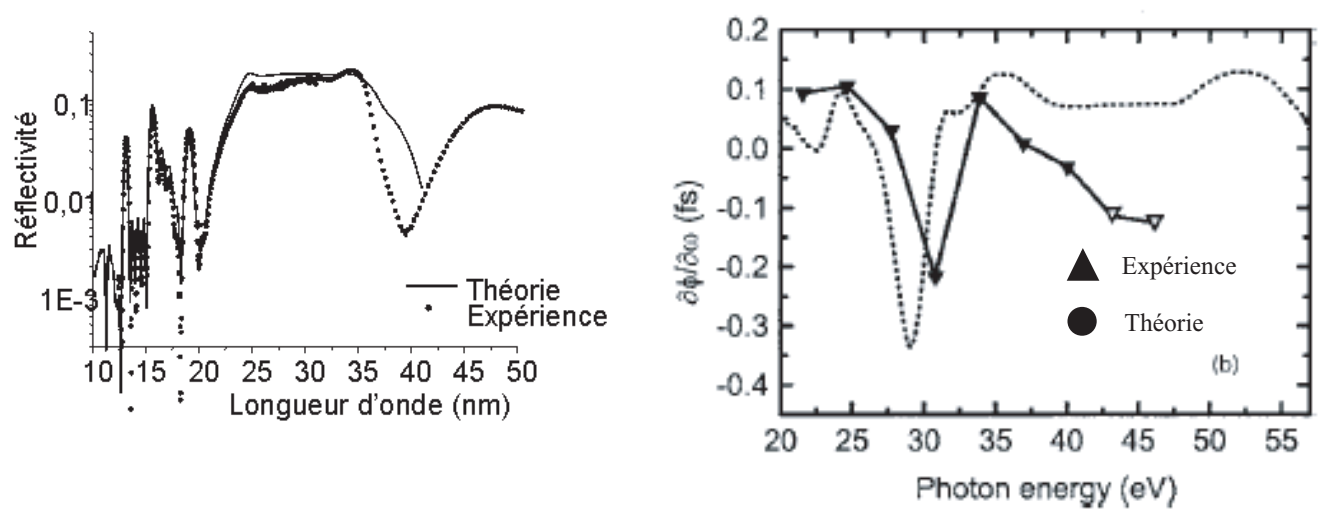

Figure 5. (a) Réflectivités expérimentales en fonction de la longueur d'onde pour la multicouche large bande. (b) Déphasage induit par la multicouche en fonction de l'énergie. 


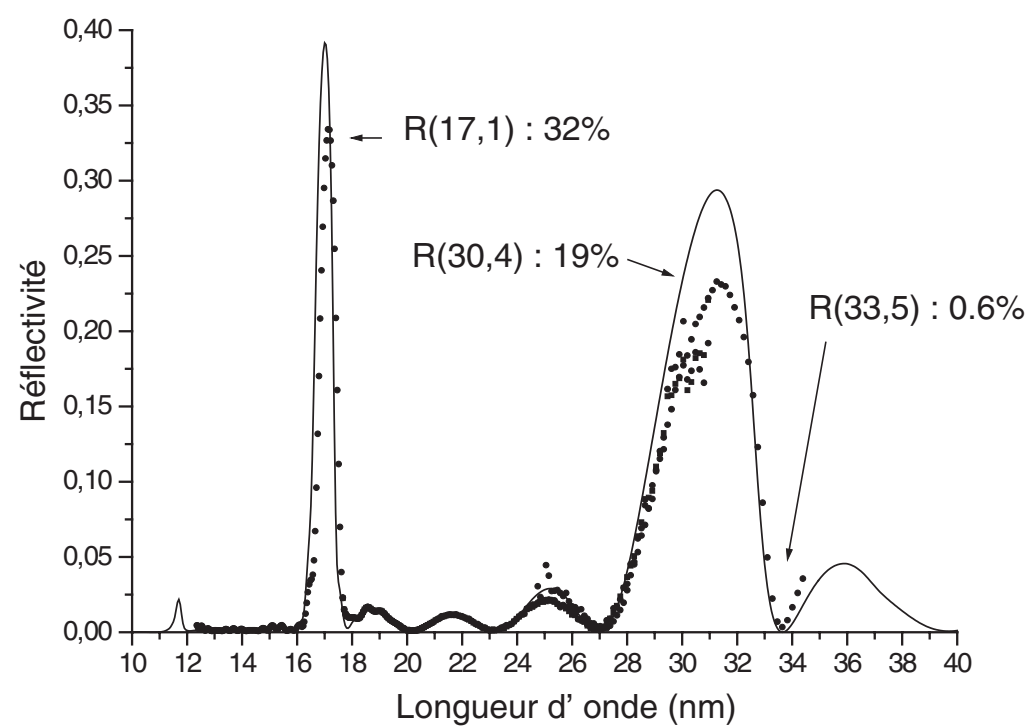

Figure 6. Réflectivités expérimentale (poins) et théorique (ligne) en fonction de la longueur d'onde pour la multicouche double bande.

couramment observées. Les longueurs d'onde choisies sont $\lambda=17,1 \mathrm{~nm}$ (Fe-IX) et $\lambda=30,4 \mathrm{~nm}$ (HeII). Cet empilement offre l'avantage de permettre l'observation de deux raies d'émission solaire à l'aide d'un seul instrument, ce qui réduit le poids de ce dernier et ainsi son coût. Cet empilement sans filtre a été mesuré au rayonnement synchrotron d'Elletra sous une incidence de $10^{\circ}$. La figure 6 montre la mesure de la réflectivité en fonction de la longueur d'onde ainsi que la courbe calculée. Les réflectivités sont respectivement de $32 \%$ à $\lambda=17,1 \mathrm{~nm}$ et de $19 \%$ à $\lambda=30,4 \mathrm{~nm}$.

\section{CONCLUSION}

Nous avons développé de nouvelles multicouches qui ouvrent de nouvelles perspectives d'applications. Nous avons développé et étudié les performances des multicouches $\mathrm{Sc} / \mathrm{Si}$. Ces multicouches possèdent des réflectivités expérimentales élevées de l'ordre de $46 \%$ à $46 \mathrm{~nm}$. Nous avons étudié la possibilité d'améliorer la stabilité thermique en ajoutant aux interfaces des couches barrières. Les matériaux que nous avons utilisé sont $\mathrm{B}_{4} \mathrm{C}, \mathrm{ScN}$. Ces études permettent de sélectionner la barrière qui possède le meilleur compromis entre la réflectivité et l'amélioration de la stabilité thermique. Par exemple une barrière de $0,3 \mathrm{~nm}$ de $\mathrm{B}_{4} \mathrm{C}$ permet d'avoir la réflectivité la plus élevée après un recuit à $200^{\circ} \mathrm{C}$. Nous avons également montré que les multicouches à trois matériaux par période peuvent être utilisées pour la réalisation de miroirs large bande. Une bande passante de $12.8 \mathrm{~nm}$ a été mesurée. Le déphasage induit par ce miroir a aussi été mesuré. Enfin nous avons réalisé un miroir multicouche permettant de réfléchir deux longueurs d'ondes spécifiques pour des applications en astrophysique.

\section{Remerciements}

Les dépôts multicouches ont été réalisés grâce à la Centrale d'Elaboration et de Métrologie d'Optiques X (CEMOX) implémentée par PraXO (Pôle d'optique des rayons X d'Orsay). Nous remercions tout particulièrement M. Idir et P. Zeitoun pour leur implication dans ce projet.

Les mesures de réflectivité ont été réalisées au rayonnement synchrotron ELLETRA sur la ligne BEAR. Nous tenons à remercier tout particulièrement A. Giglia et S. Nannarone et toute l'équipe de la ligne pour leur aide et leur soutien. 
Les mesures du déphasage induit par le miroir large bande ont été réalisées en collaboration avec l'université de Lund et le Laboratoire d'Optique Appliquée. Nous tenons à remercier toutes les personnes qui ont permis ces mesures.

Ce travail a été partiellement financé par le Centre National de la Recherche Scientifique par le programme « Nouveaux materiaux-fonctionnalités nouvelles ».

\section{Références}

[1] L. Nevot et al., Phys. Appl. 23, 1675-1686 (1988).

[2] S. Nannarone et al., dans synchrotron radiation instrumentation, Tony Warwick, John Arthur, Howard A. Padmore, Joachim Stöhr, Stanford Synchrotron, eds AIP Conference Proceedings 705, 450-453 (2004).

[3] Y.A Uspenskii et al., dans Soft X-Ray Lasers and Applications II, Jorge J. Rocca, Luiz B. Da Silva, eds Proc. SPIE 3156, pp. 288-294 (1997).

[4] N. Kaiser et al., dans Advances in Optical Thin Films, Claude Amra, Norbert Kaiser, H. Angus Macleod, eds Proc. SPIE 5250, pp. 109-118 (2003).

[5] Y. Uspenskii et al., Opt. Lett. 23, 771-773 (1998).

[6] A.V. Vinogradov et al., dans Soft X-ray Laser and Apllications IV, Ernst E. Fill, Jorge J.G Rocca, Eds Proc. SPIE 4505, pp. 230-235 (2001).

[7] A.F. Jankowski et al., Thin Solid films 469-470, 372-376 (2004).

[8] J. Gautier et al., Applied optics 44, 384 (2005).

[9] J.F. Seely et al., Applied Optics 43, 1463 (2004).

[10] P.M Paul et al., Phys. Rev. Lett. 77, 1689-1692 (1996).

[11] Y. Mairesse et al., Science 302, 1540 (2003).

[12] A.S Morlens et al., Opt. Lett. 30, 1554-1556 (2005).

[13] A.S Morlens et al., Opt. Lett. 31, 1558-1560 (2006). 